\title{
New Approach to the Ramsey Model
}

\author{
Dr. Sergei Aliukov
}

\section{Dr. Vladimir Gorshenin}

South Ural State University (Chelyabinsk, Russia); Email: alysergey@gmail.com

\section{Doi:10.5901/mjss.2015.v6n6p284}

\begin{abstract}
In this paper it is suggested a new approach to the Ramsey model with the Constant Relative Risk Aversion (CRRA) utility function and Cobb-Douglas technology. The suggested approach allows finding a steady state which is new and unknown before. It is proved that the found steady state is stable. With help of the method of a small parameter analytical solution of the model has been obtained. Some opportunities for improving the obtained analytical solution are suggested. It is shown that transversely condition, which is a component part of the Rumsey model, holds true for the new approach.
\end{abstract}

Keywords: economic growth, Ramsey model, steady-state, stability.

\section{Introduction}

The model of economic growth suggested by Ramsey (1928) and adapted by Cass (1965) and Koopmans (1968) is one of the most important theoretical construct in modern macroeconomics. This model is the basis for both growth theory and business cycle theory. It takes pride of place in an every course of Macroeconomics and Growth Theory. Many economists and scientists who do research in the field of Economics soundly believe (Mehlum 2002) that two of the main contributions to Growth Theory are the Ramsey growth model and the Solow model (Sollow 1956).

However, despite this popularity, there is a paucity of analytical solutions of the Ramsey model (Smith 2006). For instance, Long and Plosser (1983) developed a discrete-time example with log utility, Cobb-Douglas technology, and one hundred percent deprecation which is often used to exposit RBC models. Also, it is possible to derive an explicit solution when there is a constant gross saving rate (Kurz 1968; Barro at al. 1995; Barro at al. 2004; Walde 2005). Mehlum (2005) has provided a close form solution for the case of a Leontieff technology. Besides, he found an explicit solution for the Ramsey Saddle Path (Mehlum 2002). Recently, William T. Smith (2006) has found a closed form solution to the Ramsey model. In another paper William T. Smith (2005) derives an explicit solution to a stochastic version of the Ramsey model. The presence of uncertainty makes the investigation of the model much more complicted. One more paper dedicated to the model is written by Takashi Kamihigashi (2014).

The model is a system of non-linear differential equations. Usually, such kind of systems do not allow finding an exact solution in general case. However, in some special cases it is possible to do it. But in these cases we have to use some restrictions. Therefore, each such kind of the above-mentioned solutions involves some unappealing features. The assumption of one hundred percent depreciation in Long and Plosser is highly unrealistic. The same we can say about the restriction of $\log$ preferences. At any rate, this example only yields a solution in discrete time, it doesn't work in continuous time. The constant saving rate formulation requires the elasticity of intertemporal substitution to be tied to the rates of depreciation and time preference in an unintuitive way. The Leontieff technology has long been known to be a razor-edge case. In his paper Halvor Mehlum (2002) used the production function that was of the fixed coefficient type. The choice of such a kind of production function may appear to be restrictive. In William Smith's solution there is assumption that capital's share is equal to the reciprocal of the intertemporal elasticity of substitution. It is absolutely clear that this assumption reduces the value of the obtained solution in a great extent. This is admittedly a special case.

It would be nice to have an example with a reasonable rate of depreciation, a non-unitary elasticity of intertemporal substitution, and diminishing returns. Absent such a solution, empirical implementation of the model has required either a linear approximation around the steady state or numerical simulation.

This paper will derive an explicit solution to the canonical version of the Ramsey model with constant elasticity preferences and Cobb-Douglas technology. 


\section{The Ramsey Model}

This model is so familiar, therefore description of it will be succinct. Imagine an economy inhabited by a large number of identical, infinitely-lived consumers. For simplicity there is neither population growth nor technological growth. I adopt the functional forms commonly used in empirical, and in many theoretical, applications: technology is Cobb-Douglas, with a capital share of $\alpha \in[0,1]$; the rate of depreciation $\delta \in[0,1]$ is constant; there is a constant rate of time preference $\rho \in[0, \infty)$ and preferences are time-separable, with a constant elasticity of intertemporal substitution, $\sigma \in[0, \infty)$.

The dynamics of per capita consumption $c$ and capital $k$ are determined by the following system of differential equations (Smith 2006):

$$
\left\{\begin{array}{l}
\dot{k}=k^{\alpha}-\delta k-c ; \\
\frac{\dot{c}}{c}=\sigma\left(\alpha k^{\alpha-1}-\delta-\rho\right) .
\end{array}\right.
$$

The first of the equation of the system is the resource constraint. The second equation of the system is the Euler equation. Besides, there are two boundary conditions: the initial capital stock $k_{0}$ and the transversality condition

$$
\lim _{t \rightarrow \infty} e^{-\rho t} c^{-\frac{1}{\sigma}} k=0 \text {. }
$$

The steady state for the system (1) is

$$
\left\{\begin{array}{l}
\bar{k}=\left(\frac{\delta+\rho}{\alpha}\right)^{\frac{1}{\alpha-1}} ; \\
\bar{c}=\bar{k}^{\alpha}-\delta \bar{k}=\frac{\rho+\delta(1-\alpha)}{\alpha} \bar{k} .
\end{array}\right.
$$

This steady state is saddle-path type and it is unstable.

In this paper a new steady state has been found and the steady state is stable. This new steady state can be found by writing the Ramsay model (1) in a slightly modified form. Let us show it in more detail.

In the form of (1) the Ramsey growth model assumes that, as follows from the second equation of the system, $c \neq 0$. However, there is nothing stopping to write the system (1) as follows:

$$
\left\{\begin{array}{l}
\dot{k}=k^{\alpha}-\delta k-c ; \\
\dot{c}=c \sigma\left(\alpha k^{\alpha-1}-\delta-\rho\right) .
\end{array}\right.
$$

Assuming $\dot{k}=0, \dot{c}=0$, we can find the new steady state

$$
\left\{\begin{array}{l}
\bar{k}=\delta^{\frac{1}{\alpha-1}} \\
\bar{c}=0 .
\end{array}\right.
$$

It can be shown, that this steady state, in contrast to the solution (3) is asymptotically stable, satisfying the transversally condition (2).

At first glance, the found steady state (5) may seem strange, since in this position, per capita consumption is zero. On the other hand, taking limited resources into consideration, it may be that this steady state, on the contrary, the most true in the long run.

\section{An Analytical Approximate Solution}

Let us find the solution of the system (4).

The system of equations (4) is dauntingly nonlinear and despite its relative simplicity, the system does not allow an exact solution. To approach a solution we have to use approximate analytical and numerical methods. In this paper we find the approximate analytical solution of the system (4) with help of the method of a small parameter. We show how to do it. 
Functions $k^{\alpha}, k^{\alpha-1}$ we expand in Taylor series in the neighborhood of the steady state (5):

$k^{\alpha}=\bar{k}^{\alpha}+\alpha \bar{k}^{\alpha-1}(k-\bar{k})+\frac{\alpha(\alpha-1)}{2} \bar{k}^{\alpha-2}(k-\bar{k})^{2}+\ldots$;

$k^{\alpha-1}=\bar{k}^{\alpha-1}+(\alpha-1) \bar{k}^{\alpha-2}(k-\bar{k})+\frac{(\alpha-1)(\alpha-2)}{2} \bar{k}^{\alpha-3}(k-\bar{k})^{2}+\ldots$

Using the substitution $\tilde{k}=k-\bar{k}$ we get $k=\tilde{k}+\bar{k}, \dot{k}=\dot{\tilde{k}}$.

The Taylor series expansions here take the following form

$k^{\alpha}=\bar{k}^{\alpha}+\alpha \bar{k}^{\alpha-1} \tilde{k}+\frac{\alpha(\alpha-1)}{2} \bar{k}^{\alpha-2} \tilde{k}^{2}+\ldots ;$

$k^{\alpha-1}=\bar{k}^{\alpha-1}+(\alpha-1) \bar{k}^{\alpha-2} \tilde{k}+\frac{(\alpha-1)(\alpha-2)}{2} \bar{k}^{\alpha-3} \tilde{k}^{2}+\ldots$

Taking (5) into consideration, these expansions can be rewritten as

$k^{\alpha}=\delta^{\frac{\alpha}{\alpha-1}}+\alpha \delta \tilde{k}+\frac{\alpha(\alpha-1)}{2} \delta^{\frac{\alpha-2}{\alpha-1}} \tilde{k}^{2}+\ldots ;$

$k^{\alpha-1}=\delta+(\alpha-1) \delta^{\frac{\alpha-2}{\alpha-1}} \tilde{k}+\frac{(\alpha-1)(\alpha-2)}{2} \delta^{\frac{\alpha-3}{\alpha-1}} \tilde{k}^{2}+\ldots$

Substitute these expansions into the system (4) to arrive at the following system (6) after some transformations

$\left\{\begin{array}{l}\dot{\tilde{k}}+(1-\alpha) \delta \tilde{k}=\frac{\alpha(\alpha-1)}{2} \delta^{\frac{\alpha-2}{\alpha-1}} \tilde{k}^{2}+\frac{\alpha^{2}(\alpha-1)^{2}(\alpha-2)}{6 \alpha(\alpha-1)} \delta^{\frac{\alpha-3}{\alpha-1}} \tilde{k}^{3}+\ldots-c ; \\ \dot{c}+\sigma((1-\alpha) \delta+\rho) c=\sigma \alpha(\alpha-1) \delta^{\frac{\alpha-2}{\alpha-1}} \tilde{k} c+\sigma \frac{\alpha^{2}(\alpha-1)^{2}(\alpha-2)}{2 \alpha(\alpha-1)} \delta^{\frac{\alpha-3}{\alpha-1} \tilde{k}^{2} c+\ldots}\end{array}\right.$

Define the small parameter by $\mu=\alpha(\alpha-1)$. It is not difficult to show that $|\mu| \leq 0,25$. Using this transformation the system (6) can be rewritten as

$$
\left\{\begin{array}{l}
\dot{\tilde{k}}+(1-\alpha) \delta \tilde{k}=\frac{\mu}{2} \delta^{\frac{\alpha-2}{\alpha-1}} \tilde{k}^{2}+\mu^{2} \frac{(\alpha-2)}{6 \alpha(\alpha-1)} \delta^{\frac{\alpha-3}{\alpha-1} \tilde{k}^{3}+\ldots-\frac{\mu}{\alpha(\alpha-1)} c ;} \\
\dot{c}+\sigma((1-\alpha) \delta+\rho) c=\mu \sigma \delta^{\frac{\alpha-2}{\alpha-1}} \tilde{k} c+\mu^{2} \frac{\sigma(\alpha-2)}{2 \alpha(\alpha-1)} \delta^{\frac{\alpha-3}{\alpha-1}} \tilde{k}^{2} c+\ldots
\end{array}\right.
$$

The approximate analytical solution of the system (7) can be obtained in the form of series in powers of the small parameter

$$
\left\{\begin{array}{l}
\tilde{k}=\tilde{k}^{*}+\mu \tilde{k}_{1}+\mu^{2} \tilde{k}_{2}+\ldots \\
c=c^{*}+\mu c_{1}+\mu^{2} c_{2}+\ldots
\end{array}\right.
$$

where $\tilde{k}^{*}$ and $c^{*}$ correspond to the so called generating solution.

Taking into account the relations (8), the system (7) can be rewritten as

$$
\left\{\begin{array}{l}
\dot{\tilde{k}^{*}}+\mu \dot{\tilde{k}}_{1}+\mu^{2} \dot{\tilde{k}}_{2}+\ldots(1-\alpha) \delta\left(\tilde{k}^{*}+\mu \tilde{k}_{1}+\mu^{2} \tilde{k}_{2}+\ldots\right)=\frac{\mu}{2} \delta^{\frac{\alpha-2}{\alpha-1}}\left(\tilde{k}^{*}+\mu \tilde{k}_{1}+\mu^{2} \tilde{k}_{2}+\ldots\right)^{2}+ \\
+\mu^{2} \frac{(\alpha-2)}{6 \alpha(\alpha-1)} \delta^{\frac{\alpha-3}{\alpha-1}}\left(\tilde{k}^{*}+\mu \tilde{k}_{1}+\mu^{2} \tilde{k}_{2}+\ldots\right)^{3}+\ldots-\frac{\mu}{\alpha(\alpha-1)}\left(c^{*}+\mu c_{1}+\mu^{2} c_{2}+\ldots\right) ; \\
\dot{c}^{*}+\mu \dot{c}_{1}+\mu^{2} \dot{c}_{2}+\ldots+\sigma((1-\alpha) \delta+\rho)\left(c^{*}+\mu c_{1}+\mu^{2} c_{2}+\ldots\right)= \\
=\mu \sigma \delta^{\frac{\alpha-2}{\alpha-1}}\left(\tilde{k}^{*}+\mu \tilde{k}_{1}+\mu^{2} \tilde{k}_{2}+\ldots\right)\left(c^{*}+\mu c_{1}+\mu^{2} c_{2}+\ldots\right)+ \\
+\mu^{2} \frac{\sigma(\alpha-2)}{2 \alpha(\alpha-1)} \delta^{\frac{\alpha-3}{\alpha-1}}\left(\tilde{k}^{*}+\mu \tilde{k}_{1}+\mu^{2} \tilde{k}_{2}+\ldots\right)^{2}\left(c^{*}+\mu c_{1}+\mu^{2} c_{2}+\ldots\right)+\ldots
\end{array}\right.
$$

Putting in the system (9) $\mu=0$, we obtain a system of differential equations for the generating solution

$\left\{\begin{array}{l}\dot{\tilde{k}}^{*}+(1-\alpha) \delta \tilde{k}^{*}=0 ; \\ c^{*}+\sigma((1-\alpha) \delta+\rho) c^{*}=0 .\end{array}\right.$

Denote $\beta=(1-\alpha) \delta, \gamma=\sigma((1-\alpha) \delta+\rho)$, then we can write the last system in such a way 


$$
\left\{\begin{array}{l}
\dot{\hat{k}^{*}+\beta \tilde{k}^{*}}=0 \\
\dot{c^{*}+\gamma c^{*}}=0
\end{array}\right.
$$

This resulting system is linear. Solving it, we find the generating solution

$$
\left\{\begin{array}{l}
\tilde{k}^{*}=\tilde{k}_{0} e^{-\beta t} \\
c^{*}=c_{0} e^{-\gamma t}
\end{array}\right.
$$

where $\tilde{k}_{0}=\tilde{k}(t=0) ; c_{0}=c(t=0)$ are initial conditions.

To find $\tilde{k}_{1}$ and $c_{1}$, we reserve in the system (9) only the terms containing a small parameter in the first power. Then we obtain

$$
\left\{\begin{array}{l}
\dot{\tilde{k}}_{1}+\beta \tilde{k}_{1}=\frac{1}{2} \delta^{\frac{\alpha-2}{\alpha-1}} \tilde{k}^{* 2}-\frac{1}{\alpha(\alpha-1)} c^{*} ; \\
\dot{c}_{1}+\gamma c_{1}=\sigma \delta^{\frac{\alpha-2}{\alpha-1}} \tilde{k}^{*} c^{*} .
\end{array}\right.
$$

Substituting the generating solution found earlier into the resulting system, we get

$$
\left\{\begin{array}{l}
\dot{\tilde{k}}_{1}+\beta \tilde{k}_{1}=P_{1} e^{-2 \beta t}+P_{2} e^{-\gamma t} \\
\dot{c}_{1}+\gamma c_{1}=P_{3} e^{-(\beta+\gamma) t}
\end{array}\right.
$$

where

$$
P_{1}=\frac{1}{2} \tilde{k}_{0}^{2} \delta^{\frac{\alpha-2}{\alpha-1}} ; P_{2}=\frac{c_{0}}{\alpha(1-\alpha)} ; P_{3}=\sigma \tilde{k}_{0} c_{0} \delta^{\frac{\alpha-2}{\alpha-1}} .
$$

The system (10) is linear. Solving it, we obtain

$$
\left\{\begin{array}{l}
\tilde{k} \approx \tilde{k}^{*}+\alpha(\alpha-1) \tilde{k}_{1} ; \\
c \approx c^{*}+\alpha(\alpha-1) c_{1} .
\end{array}\right.
$$

Taking only the first two terms in the expansions (8), we obtain the final solution of the system (6), and hence the system (4)

$$
\begin{aligned}
& \left\{\begin{array}{l}
\tilde{k}_{1}=\left(P_{4}+P_{5}\right) e^{-\beta t}-P_{4} e^{-2 \beta t}-P_{5} e^{-\gamma t} ; \\
c_{1}=P_{6}\left(e^{-\gamma t}-e^{-(\beta+\gamma) t}\right),
\end{array}\right. \\
& P_{4}=\frac{\tilde{k}_{0}^{2}}{2(1-\alpha)} \delta^{\frac{1}{1-\alpha}} ; P_{5}=\frac{c_{0}}{\alpha(\alpha-1)(\beta-\gamma)} ; P_{6}=\frac{\sigma \tilde{k}_{0} c_{0}}{1-\alpha} \delta^{\frac{1}{1-\alpha}} .
\end{aligned}
$$

Taking only the first two terms in the expansions (8), we obtain the final solution of the system (6), and hence the system (4)

$$
\begin{aligned}
& \left\{\begin{array}{l}
\tilde{k} \approx\left(\tilde{k}_{0}-A_{1}-A_{2}\right) e^{-\beta t}+A_{1} e^{-2 \beta t}+A_{2} e^{-\gamma t} ; \\
c \approx\left(c_{0}-B\right) e^{-(\beta+\gamma) t},
\end{array}\right. \\
& \text { here } A_{1}=\frac{\alpha}{2} \tilde{k}_{0}^{2} \delta^{\frac{1}{1-\alpha}} ; A_{2}=\frac{c_{0}}{\gamma-\beta} ; B=\alpha \sigma \tilde{k}_{0} c_{0} \delta^{\frac{1}{1-\alpha}} .
\end{aligned}
$$

Taking the substitution $\tilde{k}=k-\bar{k}$ into consideration, finally we get

$$
\left\{\begin{array}{l}
k \approx \delta^{\frac{1}{1-\alpha}}+\left(\tilde{k}_{0}-A_{1}-A_{2}\right) e^{-\beta t}+A_{1} e^{-2 \beta t}+A_{2} e^{-\gamma t} ; \\
c \approx\left(c_{0}-B\right) e^{-(\beta+\gamma) t},
\end{array}\right.
$$

where $\tilde{k}_{0}=k_{0}-\delta^{\frac{1}{\alpha-1}} ; k_{0}=k(t=0)$ is initial condition.

Note that there is a good opportunity to find further terms in the expansions (8) sequentially improving the obtained 
solution of the system (4). Thus, we can get the system of differential equations for the terms $\tilde{k}_{2}$ and $c_{2}$, leaving in the equations of the system (9) only the terms containing the small parameter in the second power. In this case, the resulting system is as follows

$$
\begin{aligned}
& \left\{\begin{array}{l}
\dot{\tilde{k}}_{2}+\beta \tilde{k}_{2}=L_{1} \tilde{k}^{*} \tilde{k}_{1}+L_{2} \tilde{k}^{* 3}-\frac{c_{1}}{\alpha(\alpha-1)} ; \\
\dot{c}_{2}+\gamma c_{2}=\sigma L_{1}\left(\tilde{k}^{*} c_{1}+c^{*} \tilde{k}_{1}\right)+3 \sigma L_{2} \tilde{k}^{* 2} c^{*},
\end{array}\right. \\
& L_{1}=\delta^{\frac{\alpha-2}{\alpha-1}} ; L_{2}=\frac{\alpha-2}{6 \alpha(\alpha-1)} \delta^{\frac{\alpha-2}{\alpha-1}} .
\end{aligned}
$$

Similarly, we can get the system for the terms $\tilde{k}_{3}$ and $c_{3}$. This system is as follows

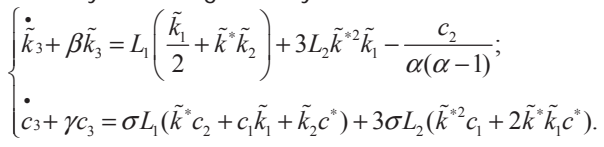

The systems (12) and (13) are linear as well, therefore if we want we can find their solutions improving the obtained solution (11) .

Numerical verification of the obtained solution that was done with help of Runge-Kutta method in a computer environment MathCAD showed good convergence of the got results. This is depicted in Figure 1.

In this figure, curves 1 correspond to per capita capital $k$ and curves 2 correspond to per capita consumption $c$. Here, solid lines are the numerical solutions and dash lines are the analytical solutions which were got with help of the method of small parameter. As shown in Figure 1, the differences are insignificant.

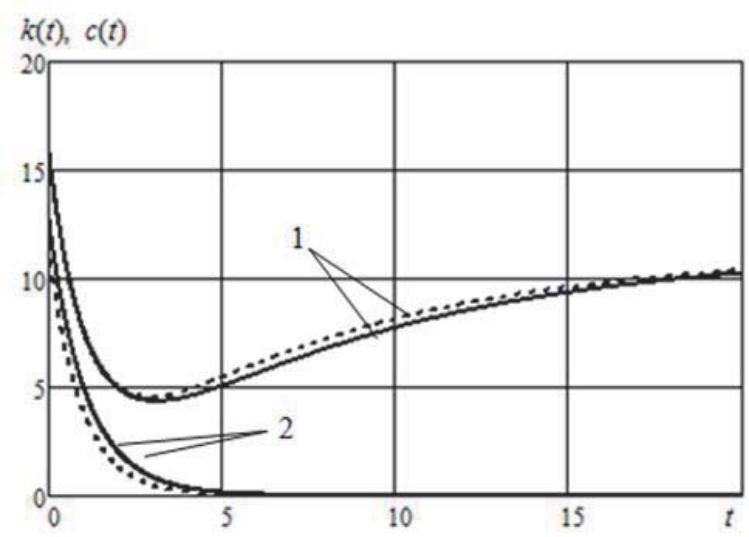

Figure 1. Graphs of per capita capital $k$ and consumption $c$

Let us check the transversality condition (2).

Because $\rho \geq 0 ; \sigma>0 ; \lim _{t \rightarrow+\infty} c=0 ; \lim _{t \rightarrow+\infty} k=\delta^{\frac{1}{\alpha-1}} \equiv$ const, we have $\lim _{t \rightarrow \infty} e^{-\rho t} c^{-\frac{1}{\sigma}} k=0$.

Let $y(t)$ be $e^{-\rho t} c^{-\frac{1}{\sigma}} k$. Then, as shown in Figure 2, $y(t) \underset{t \rightarrow \infty}{\longrightarrow} 0$. 


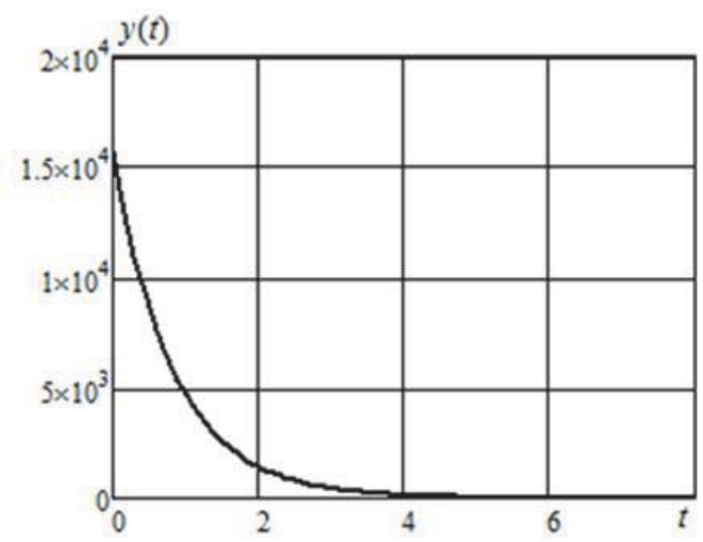

Figure 2. Transversality condition

Let us show that the obtained solution for steady state (5) is stable. We consider the autonomous system of differential equations (4). In the study of the stability, instead of the original one, we can investigate the linearized system that we can get using the system (6)

$$
\left\{\begin{array}{l}
\dot{\tilde{k}}=(\alpha-1) \delta \tilde{k}-c ; \\
\dot{c}=\sigma((\alpha-1) \delta+\rho) c,
\end{array}\right.
$$

where $\tilde{k}=k-\bar{k}=k-\delta^{\frac{1}{\alpha-1}}$.

The system (14) is the system of equations of the first approximation with respect to the original system.

The Jacobian $J$ is determined by the matrix

$$
J=\left[\begin{array}{lc}
(\alpha-1) \delta-\lambda & -1 \\
0 & \sigma((\alpha-1) \delta-\rho)-\lambda
\end{array}\right] .
$$

We find the eigenvalues of the Jacobian matrix $\mathrm{J}$ :

$$
\begin{aligned}
& \left|\begin{array}{lc}
(\alpha-1) \delta-\lambda & -1 \\
0 & \sigma((\alpha-1) \delta-\rho)-\lambda
\end{array}\right|=0 ; \\
& \lambda^{2}-((\alpha-1) \delta+\sigma((\alpha-1) \delta-\rho)) \lambda+\sigma((\alpha-1) \delta-\rho)(\alpha-1) \delta=0 ; \\
& \lambda_{1}=(\alpha-1) \delta ; \lambda_{2}=\sigma((\alpha-1) \delta-\rho) . \\
& \text { Because } \alpha \in(0,1), \delta>0, \rho>0, \sigma>0, \text { we made the conclusion } \\
& \lambda_{1}<0, \lambda_{2}<0 .
\end{aligned}
$$

According to Lyapunov theorem on stability in the first approximation if all eigenvalues $\lambda_{i}$ have negative real parts, then the steady state position of the original (4) and linearized (14) systems is asymptotically stable. This equilibrium point is a stable node.

\section{Conclusions}

This paper provides an explicit solution to the Ramsey model. It is suggested a completely new approach to the model. This approach allows us to find a new steady state which was unknown before. This finding expands the possibilities of the Ramsey model making the model more modern and useful. It is shown that the found steady state is stable.

Moreover, with help of the method of a small parameter the explicit approximate solution of the Ramsey model with the new approach is found. The error of the solution is small enough that was proved with comparing with almost exact 
numerical solution. The found solution allows getting results relatively to consumption and capital in cases with certain values of parameters involved in the model.

\section{References}

Barro, Robert, \& Xavier Sala-i-Martin (2004). Economic Growth, 2nd edition, M.I.T. Press, Cambridge.

Barro, Robert, N. Gregory Mankiw, \& Xavier Sala-i-Martin (1995). Capital Mobility in Neoclassical Models of Growth. American Economic Review, 85, 103-115.

Cass, David (1965). Optimum Growth in an Aggregative Model of Capital Accumulation. Review of Economic Studies, 32, 233-240.

Kamihigashi, Takashi (2014). Multiple Interior Steady States in the Ramsey Model with Elastic Labor Supply. Working paper series. IPAG Business School, France.

Koopmans, Tjalling (1968). On the Concept of Economic Development, in The Economic Approach to Development Planning. Elsevier, Amsterdam.

Kurz, Mordechai (1968). The General Instability of a Class of Compertitive Growth Models. Review of Economic Studies, 35, 155-174.

Long, John B., \& Charles Plosser (1983). Real Business Cycle. Journal of Political Economy, 91(1), 36-69.

Mehlum, Halvor (2005). A Closed Form Ramsey Saddle Path. Contribution to Macroeconomics, 5(1), 1-15.

Mehlum, Halvor (2002). At Last! An Explicit Solution for the Ramsey Saddle Path. Memorandum, Department of Economics, University of Oslo, 26.

Ramsey, Frank P. (1928). A Mathematical Theory of Saving. Economic Journal, 38, 543-559.

Smith, William T. (2006) A Closed Form Solution to the Ramsey Model. Contributions to Macroeconomics, 6(1), 1-27.

Smith, William T (2005). Inspecting the Mechanism Exactly: A Closed Form Solution to the Stochastic Growth Model. Working Paper.

Sollow, Robert M (1956). A Contribution to the Theory of Economic Growth. Quarterly Journal of Economics, 70, 65-94.

Walde, Klaus (2005). Endogenous Growth Cycles. International Economic Review, 46(3), 867-894. 